\title{
Septic pulmonary embolism originated from subcutaneous abscess after living donor liver transplantation: a pitfall of postoperative management
}

\author{
Kazuhisa Takeda $\cdot$ Kuniya Tanaka $\cdot$ Takafumi Kumamoto $\cdot$ \\ Kazunori Nojiri $\cdot$ Ryutaro Mori $\cdot$ Koichi Taniguchi · \\ Ryusei Matsuyama $\cdot$ Hideaki Kato $\cdot$ Itaru Endo
}

Received: 21 January 2013/Accepted: 6 June 2013/Published online: 8 October 2013

(C) The Author(s) 2013. This article is published with open access at Springerlink.com

\begin{abstract}
The use of immunosuppressants after liver transplantation (LT) is associated with postoperative complications, including infections. A 49-year-old male underwent living-donor (LD) LT because of primary sclerosing cholangitis. He was treated with tacrolimus, mycophenolate mofetil, and steroids as immunosuppressants, discharged on postoperative day (POD) 40, and re-admitted because of severe acute cellular rejection on POD 48. Three courses of steroid pulse therapy were performed, and continuous
\end{abstract}

K. Takeda $(\bowtie) \cdot$ K. Tanaka $\cdot$ T. Kumamoto $\cdot$ K. Nojiri $\cdot$

R. Mori · K. Taniguchi · R. Matsuyama · I. Endo Department of Gastroenterological Surgery, Yokohama City

University Graduate School of Medicine, 3-9 Fukuura,

Kanazawa-ku, Yokohama 236-0004, Japan

e-mail: kazutake@yokohama-cu.ac.jp

K. Tanaka

e-mail: ktrj112@urahp.yokohama-cu.ac.jp

T. Kumamoto

e-mail: bearbook21@yahoo.co.jp

K. Nojiri

e-mail: noji1192@yokohama-cu.ac.jp

R. Mori

e-mail: ryutaromori@gmail.com

K. Taniguchi

e-mail: t1006koichi@yahoo.co.jp

R. Matsuyama

e-mail: ryusei@terra.dti.ne.jp

I. Endo

e-mail: endoit@med.yokohama-cu.ac.jp

H. Kato

Department of Internal Medicine and Clinical Immunology,

Yokohama City University Graduate School of Medicine,

Yokohama, Japan

e-mail: ekato@mac.com peripheral intravenous drip infusion therapy via the left forearm was necessary for 20 days because of appetite loss. The patient was discharged on POD 83, but re-admitted on POD 87 with pyrexia. A subcutaneous abscess was present at a puncture wound on the left forearm formed by an intravenous drip during the last hospital stay. Furthermore, computed tomography showed five pieces of cavitary or wedge-shaped nodules in the bilateral lung. Because sputum revealed the presence of Gram-positive coccus, and subcutaneous abscess and blood cultures revealed Staphylococcus aureus, the pathogenesis was septic pulmonary embolism (SPE) secondary to $S$. aureus septicemia originating from a subcutaneous abscess formed by an intravenous drip. The patient was treated with drainage of the subcutaneous abscess and antibiotic therapy, and recovered immediately. Although there have been few reports of SPE after LDLT, SPE is fatal in up to $13.3 \%$ of patients. Early diagnosis, drainage of the infectious source, and appropriate use of antimicrobial therapy should be necessary to overcome SPE. Furthermore, the identical intravenous catheters should be removed whenever possible to avoid infectious complications including SPE for patients who receive steroid pulse therapy after LDLT.

Keywords Septic pulmonary embolism $\cdot$ Living donor liver transplantation - Steroid pulse therapy
Abbreviations
LT Liver transplantation
LD Living donor
POD Postoperative day
SPE Septic pulmonary embolism
CRP C-reactive protein
ALT Alanine aminotransferase
CT Computed tomography
MRSA Methicillin-resistant Staphylococcus aureus 


\section{Introduction}

Liver transplantation (LT) is regarded as a reliable treatment for patients with end-stage liver disease [1, 2]; however, because of the need for immunosuppressants, various postoperative complications are common including infection [3]. Among the possible infectious complications after LT, septic pulmonary embolism (SPE) is a rare pathogenesis. SPE is characterized by fever, respiratory symptoms, and lung infiltrates, and is associated with extrapulmonary infections [4, 5]. SPE is serious condition because it can lead to the development of lung abscesses and empyema [5]. Here we report on a patient with SPE originating from a subcutaneous abscess following livingdonor (LD) LT. The course of this case has important implications for postoperative management after LDLT.

\section{Case report}

A 49-year-old male underwent LDLT because of primary sclerosing cholangitis. Preoperative performance score was 1 and the model for end-stage liver disease score was 24.8. The patient had a history of total colectomy because of ulcerative colitis. The donor was the patient's wife, and the blood types were identical. A right hemiliver graft was used. Graft size was $750 \mathrm{~g}$ and the ratio of graft volume/ standard liver volume of the recipient was $52.1 \%$. The cold and warm ischemic time was 111 and $56 \mathrm{~min}$, respectively. Duct-to-duct anastomosis was adopted for biliary reconstruction because postoperative management of strictures is easy to perform by endoscopic methods. Splenectomy was also performed because of preoperative thrombocytopenia resulting from splenomegaly. Expected amount of bleeding was $29,876 \mathrm{ml}$. He was treated with tacrolimus, mycophenolate mofetil, and steroids as immunosuppressants. The whole-blood trough level of tacrolimus was adjusted to $10-12 \mathrm{ng} / \mathrm{ml}$ during the first postoperative week, and was tapered thereafter. The mycophenolate mofetil doses ranged from 500 to $1,000 \mathrm{mg} /$ day. Methylprednisolone (700 mg) was administered twice intraoperatively, tapered gradually thereafter, and discontinued on postoperative day (POD) 35. The patient was discharged on POD 40.

He was re-admitted on POD 48 because of severe acute cellular rejection. After three courses of steroid pulse therapy using methylprednisolone (each comprising $500 \mathrm{mg}$ per session for 3 days; total dose of $4.5 \mathrm{~g}$ of methylprednisolone) were performed, the liver dysfunction gradually improved (Fig. 1); however, continuous peripheral intravenous drip infusion therapy via the left forearm was necessary for 20 days because of appetite loss. The patient was discharged on POD 83; however, he visited our hospital on POD 87 complaining of pyrexia accompanying pain in the left forearm. Laboratory results showed a peripheral white blood cell count of $4,000 / \mathrm{mm}^{3}$ with $22.0 \%$ stab cells, C-reactive protein (CRP) levels of $10.3 \mathrm{mg} / \mathrm{dl}$ and albumin level of $2.4 \mathrm{~g} / \mathrm{dl}$. Liver function tests, including alanine aminotransferase (ALT) measurements, were almost within the normal range (Fig. 1). Rubefaction accompanying the
Fig. 1 Postoperative clinical course showing ALT and CRP levels, and tacrolimus trough level. Treatment courses and hospital admission periods are also shown

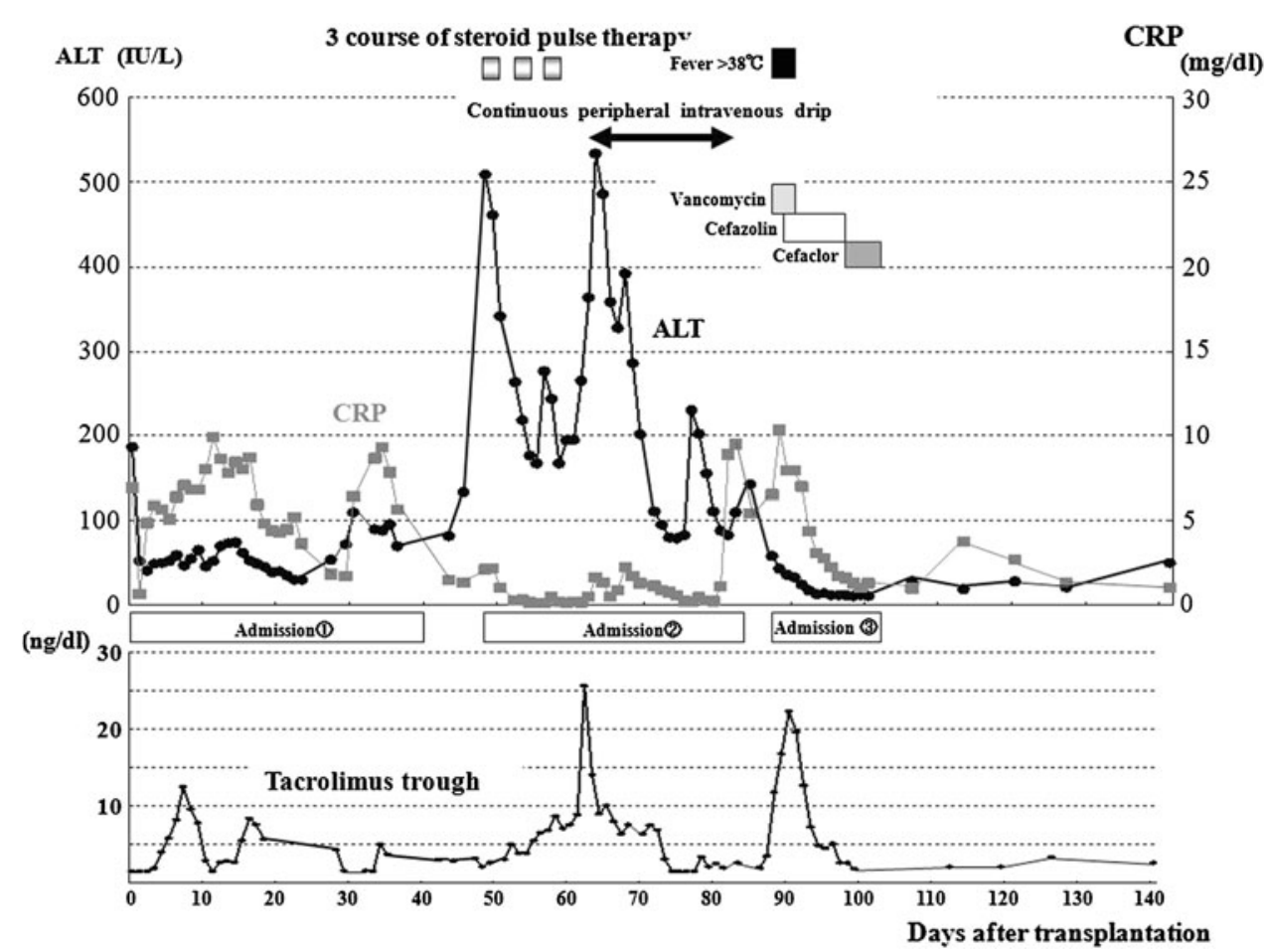


subcutaneous abscess was present at a wound on the left forearm formed by intravenous drip therapy during the last hospital stay (Fig. 2). Although respiratory symptoms were not present and performance status was zero, chest computed tomography (CT) revealed five pieces of cavitary or wedgeshaped nodules in the periphery of the bilateral lung (Fig. 3). The lung nodules ranged from $0.8-2 \mathrm{~cm}$ in diameter, and were indistinct on a chest X-ray. $\beta$-D glucan levels were $<6.0 \mathrm{pg} / \mathrm{ml}$, Aspergillus antigens were not detected and mycobacterial culture of sputum was negative. The smear from the incisional drainage of the skin, blood and sputum revealed the presence of Gram-positive coccus; empiric broad-spectrum intravenous antibiotics using vancomycin

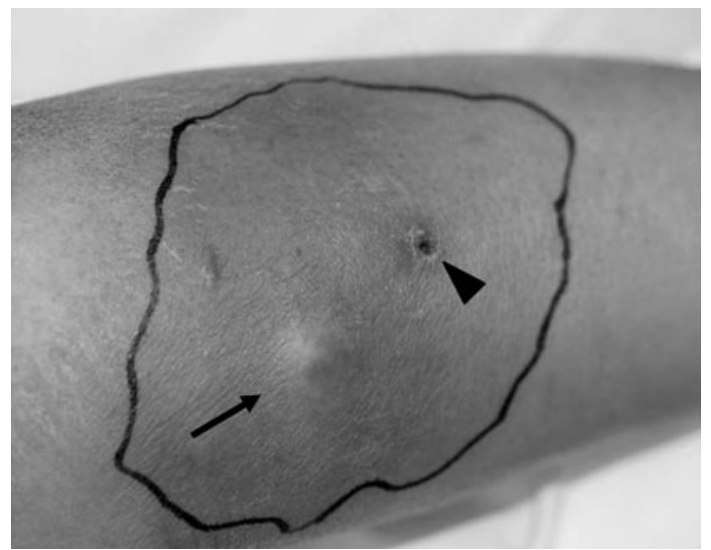

Fig. 2 Rubefaction accompanying a subcutaneous abscess (arrow) was present at the wound on the left forearm (arrowhead) formed by intravenous drip therapy during the last hospital stay
( $2 \mathrm{~g} /$ day) were thus started immediately. Lastly, methicillinsensitive Staphylococcus aureus was cultured both from the blood and the subcutaneous abscess of the left forearm.

The pathogenesis was diagnosed as SPE secondary to $S$. aureus septicemia originating from the subcutaneous abscess of the left forearm. Vancomycin was de-escalated to cefazolin $(6 \mathrm{~g} /$ day $)$ for 11 days following cefaclor treatment $(1.5 \mathrm{~g} /$ day $)$ for 4 days with drainage of the subcutaneous abscess. The patient showed a good response to the antibiotic therapy; blood cultures detected no bacteria, the subcutaneous abscess in the left forearm epithelized, and the lung cavitary lesions disappeared by 141 days after LDLT.

\section{Discussion}

SPE is defined as the coexistence of multiple lung infiltrates coupled with the isolation of bacteria from the blood and their infection source [6,7]. SPE occurs when a thrombus containing bacteria travels to the lung and reaches the pulmonary arteries $[4,8]$. Although it is a rare disorder [9], SPE is a serious infectious complication that can lead to the development of lung abscesses or bronchopleural fistulae. Moreover, SPE is fatal in up to $13.3 \%$ of patients $[5,10]$. Therefore, early diagnosis and the immediate institution of aggressive antimicrobial treatment are required to overcome SPE [11, 12].

SPE diagnosis is made in the presence of the following classic features-febrile illness, pulmonary infiltrates, and
Fig. 3 Chest CT showing five pieces of cavitary or wedgeshaped nodules in the bilateral area periphery (arrows)

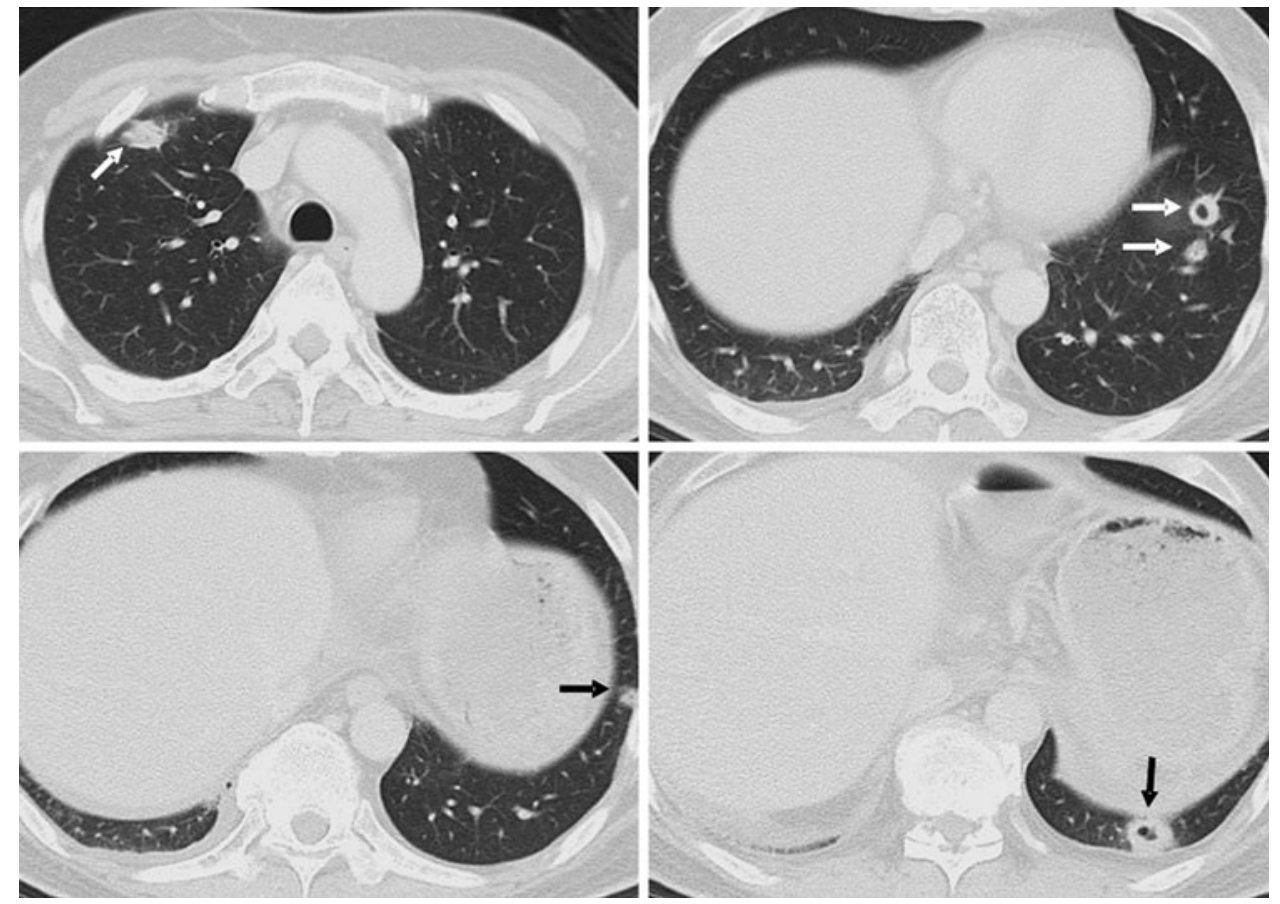


an extrapulmonary source of infection. Typical respiratory symptoms of SPE include pleuritic chest pain, cough, and hemoptysis $[4,5]$. However, respiratory complaints are not always observed [7], especially in immunosuppressed patients [5]. In a chest X-ray, the peripheral and wedgeshaped densities may be suggestive, but in most cases, these findings are not specific [13] and visible. On the other hand, CT is a more sensitive diagnostic test than chest $\mathrm{X}$-ray [14]. Therefore, we recommend that chest CT scans should be performed in the presence of clinical features accompanying the isolation of bacteria from the blood and the infectious focus, especially in patients with SPE risk factors such as described below.

The risk factors leading to SPE include intravenous drug abuse, pelvic thrombophlebitis, an immunocompromised state, head and neck infections, endocarditis, and indwelling intravenous catheters $[4,5]$. In the patient reported here, 20-day peripheral continuous intravenous drip therapy had to be performed because of appetite loss during steroid pulse therapy. The identical intravenous catheter had been used during this clinical course. The optimal duration of use of the identical intravenous catheters is controversial [15], but they should be removed or exchanged earlier to avoid infectious complications including SPE for patients who receive steroid pulse therapy after LDLT.

SPE progresses to a lung abscess rapidly $[8,9]$. In a lung abscess, a localized parenchymal infection becomes necrotic [16], an abscess with air/fluid level is identified in the lung cavity [8, 16], the sputum is purulent and, on Gram staining, many bacteria and polymorphonuclear leulocytes are detected. In the present case, no abscess formation was detected in the lung cavities by $\mathrm{CT}$ and the sputum was not purulent. Therefore, the pathogenesis was SPE and did not progress to lung abscess.

SPE treatment depends on identifying the source of infection, and choosing the appropriate antimicrobial therapy [5] at an early stage. One pathogen commonly associated with SPE is $S$. aureus $[4,9,12]$, and methicillinresistant S. aureus (MRSA) strains are often reported [4, 9]. In the current patient, vancomycin was used as an empiric therapy until the pathogen was identified, as MRSA infections are common after LDLT [17]. However, vancomycin is not recommended for long-term use because of the selection risk of vancomycin-resistant $S$. aureus. The recently uploaded Surviving Sepsis Campaign Guidelines recommend a treatment strategy involving the use of a broad-spectrum, empirical antimicrobial regimen, followed by a de-escalated, pathogen-specific therapy to lessen the risk of developing antibiotic resistance $[18,19]$. Based on these guidelines, we replaced vancomycin with cefazolin as soon as the pathogen was identified as methicillin-sensitive $S$. aureus in the current patient. Furthermore, when infectious disease occurred after LDLT, the trough level of tacrolimus had to be controlled to prevent excessive immunosuppression. In the present patient, tacrolimus was administered orally and target trough level was $2 \mathrm{ng} / \mathrm{ml}$. However, there were two episodes of over-dosing of tacrolimus during the clinical course, because the patient sometimes had diarrhea after total colectomy. Further strict monitoring of immunosuppressants had to be necessary.

To the best of our knowledge, only one other patient has been reported to suffer from SPE after LDLT [20]; however, SPE remains one of the more serious infectious complications in immunosuppressed patients. Early diagnosis, drainage of the infection source, and appropriate use of antimicrobial therapy should be necessary to overcome SPE. Furthermore, the identical intravenous catheters should be removed whenever possible to avoid infectious complications including SPE for patients who receive steroid pulse therapy after LDLT.

Conflict of interest The authors declare that they have no conflict of interest.

Open Access This article is distributed under the terms of the Creative Commons Attribution License which permits any use, distribution, and reproduction in any medium, provided the original author(s) and the source are credited.

\section{References}

1. Hong SK, Hwang S, Lee SG, Lee LS, Ahn CS, Kim KH, et al. Pulmonary complications following adult liver transplantation. Transplant Proc. 2006;38:2979-81.

2. Yoshimura N, Okajima H, Ushigome H, Sakamoto S, Fujiki M, Okamoto M. Current status of organ transplantation in Japan and worldwide. Surg Today. 2010;40:514-25.

3. Takeda K, Morioka D, Kumamoto T, Matsuo K, Tanaka K, Endo I, et al. A survival case of ABO-incompatible liver transplantation complicated with severe preoperative infection and subsequent overwhelming postsplenectomy infection. Transplant Proc. 2009;41:3941-4.

4. Kruse BT, Vadeboncoeur TF. Methicillin-resistant Staphylococcus aureus sepsis presenting with septic pulmonary emboli. J Emerg Med. 2009;37:383-5.

5. Cook RJ, Ashton RW, Aughenbaugh GL, Ryu JH. Septic pulmonary embolism: presenting features and clinical course of 14 patients. Chest. 2005;128:162-6.

6. Tragiannidis A, Tzouvelekis G, Eboriadou M, Fidani L, Pavlou E, Papageorgiou T, et al. Septic pulmonary embolism due to Staphylococcus aureus. Pediatr Int. 2008;50:717.

7. Yüksel H, Ozgüven AA, Akil I, Ergüder I, Yilmaz D, Cabuk M. Septic pulmonary emboli presenting with deep venous thrombosis secondary to acute osteomyelitis. Pediatr Int. 2004;46: 621-3.

8. Wong KS, Lin TY, Huang YC, Hsia SH, Yang PH, Chu SM. Clinical and radiographic spectrum of septic pulmonary embolism. Arch Dis Child. 2002;87:312-5.

9. Celebi S, Hacimustafaoglu M, Demirkaya M. Septic pulmonary embolism in a child. Indian Pediatr. 2008;45:415-7. 
10. MacMillan JC, Milstein SH, Samson PC. Clinical spectrum of septic pulmonary embolism and infarction. J Thorac Cardiovasc Surg. 1978;75:670-9.

11. Sözener ZC, Kayal A, Atasoy C, Kilickap M, Numanoglu N, Savas I. Septic pulmonary embolism: three case reports. Monaldi Arch Chest Dis. 2008;69:75-7.

12. McKerlie I, Bloom C, Kreisman H. Staphylococcus aureus septic pulmonary emboli presenting as angioinvasive aspergillosis: case report. Can Assoc Radiol J. 2001;52:395-8.

13. Huang RM, Naidich DP, Lubat E, Schinella R, Garay SM, McCauley DI.Septic pulmonary emboli: CT-radiographic correlation. Am J Roentgenol. 1989 (1):41-5.

14. Iwasaki Y, Nagata K, Nakanishi M, Natuhara A, Harada H, Kubota Y, et al. Spiral CT findings in septic pulmonary emboli. Eur J Radiol. 2001;37:190-4.

15. Rickard CM, McCann D, Munnings J, McGrail MR. Routine resite of peripheral intravenous devices every 3 days did not reduce complications compared with clinically indicated resite: a randomised controlled trial. BMC Med. 2010;8:53.

16. Puligandla PS, Laberge JM. Respiratory infections: pneumonia, lung abscess, and empyema. Semin Pediatr Surg. 2008;17(1): 42-52.

17. Matsuo K, Sekido H, Morioka D, Sugita M, Nagano Y, Takeda $\mathrm{K}$, et al. Surveillance of perioperative infections after adult living donor liver transplantation. Transpl Proc. 2004;36:2299-301.
18. Shime N, Satake S, Fujita N. De-escalation of antimicrobials in the treatment of bacteraemia due to antibiotic-sensitive pathogens in immunocompetent patients. Infection. 2011;39:319-25.

19. Dellinger RP, Levy MM, Carlet JM, Bion J, Parker MM, Jaeschke $\mathrm{R}$, et al. International Surviving Sepsis Campaign Guidelines Committee; American Association of Critical-Care Nurses; American College of Chest Physicians; American College of Emergency Physicians; Canadian Critical Care Society; European Society of Clinical Microbiology and Infectious Diseases; European Society of Intensive Care Medicine; European Respiratory Society; International Sepsis Forum; Japanese Association for Acute Medicine; Japanese Society of Intensive Care Medicine; Society of Critical Care Medicine; Society of Hospital Medicine; Surgical Infection Society; World Federation of Societies of Intensive and Critical Care Medicine. Surviving Sepsis Campaign: international guidelines for management of severe sepsis and septic shock: 2008. Crit Care Med 2008;36: 296-327.

20. Avery RK, Barnes DS, Teran JC, Wiedemann HP, Hall G, Wacker $\mathrm{T}$, et al. Listeria monocytogenes tricuspid valve endocarditis with septic pulmonary emboli in a liver transplant recipient. Transpl Infect Dis. 1999;1:284-7. 